\title{
Inventory and Spatial Assemblage Study of Reef Fish in the Area of Andavadoaka, South-West Madagascar (Western Indian Ocean)
}

\author{
C.J. Gillibrand ${ }^{1}$, A.R. Harris ${ }^{1 *}$ and E. Mara ${ }^{2}$ \\ ${ }^{I}$ Blue Ventures Conservation, 52 Avenue Road, London, N6 5DR, UK, ${ }^{2}$ Institut Halieutique et des Sciences \\ Marines (IH.SM), B.P. 141 - Route du Port, Toliara 601, Madagascar
}

\begin{abstract}
Keywords: Visual census, reef fish assemblage, Andavadoaka, Madagascar, Western Indian Ocean
\end{abstract}

\begin{abstract}
This study represents the first qualitative census of the reef fish assemblage of coral reefs in the vicinity of Andavadoaka, south-west Madagascar. Observational data of fish species sightings were collected over the course of a year, while visual census data, with low observer bias, detailing fish assemblage from the four major reef zones of the area were collected in a shorter 5-week period. A total of three hundred and thirty four (334) species from 58 families were recorded throughout the year, while one hundred and eighty seven (187) species were observed during the shorter assemblage study. The trophic habits of the fish population are comparable with other sites in the Western Indian Ocean, and suggest a healthy fish community, despite evidence of broad-scale damage from coral bleaching and associated mortality. The authors suggest that this can be explained by current relatively low levels of anthropogenic impacts from fishing. However, given the extent of regional reef degradation, fish species diversity may now be particularly vulnerable to future anthropogenic or climatic disturbances, such as increased fishing effort or renewed coral bleaching episodes.
\end{abstract}

\section{INTRODUCTION}

Although there have been a number of studies detailing fish populations in Madagascar (Maugé 1967; Harmelin-Vivien 1977; Harmelin-Vivien 1979), few have been undertaken in the last 3 decades, and no such studies have been carried out in the remote northern areas of the large emergent reef system of the South-West of Madagascar. This extensive reef system stretches from Androka in the south to Morombe in the north, a total distance of some $458 \mathrm{~km}$ (Cooke et al. 2000). In the region of Toliara, the reef forms a large barrier (the Grand Récif de Tulear), while further to the north this barrier fragments, comprising a series of patch reefs and fringing reefs, the latter found both adjacent to the coast and surrounding barrier islands and sand cays.

The region's southern reefs were studied in the 1970s (Clausade et al. 1971; Pichon, 1972; Battistini, 1960), although the majority of research focussed on the physical structure and benthic communities of the reefs (Pichon, 1974). Of the few studies of reef fish that have been undertaken in the area, almost all have been restricted to the Grand Récif de Tulear (Maugé, 1967; HarmelinVivien, 1977; Harmelin-Vivien, 1979). Coral reefs throughout the region provide a vital resource base for local traditional and artisanal fisheries.

Marine biodiversity is threatened throughout the world's oceans (Bellwood et al. 2004; Sala \& Nnowlton 2006; Worm et al. 2006). From a biogeographical perspective, marine diversity 
checklists are critical to identifying geographical variations in species distributions between regions. This knowledge forms a fundamental precursor to understanding processes regulating marine biodiversity, and predicting potential consequences of biodiversity loss to ecosystem function (Bellwood \& Hughes 2001). In the Indo-Pacific province such diversity assessments provide a means of understanding longitudinal and latitudinal gradients of species richness away from the Indo-Philippines 'centre' of marine biodiversity (Hughes et al. 2002). Coral reef fish species inventories for the western Indian Ocean region are limited in their coverage (Chabanet \& Durville 2005) and no data have yet been published detailing reef fish assemblages for the northern areas of the south-west Madagascar reef system. Of particular importance to diversity assessments in the region is a better understanding of faunal differences between the extensive coral reefs of south-west Madagascar and other sites in the south-west Indian Ocean. This study aims to address this gap in published records, presenting research from Andavadoaka to provide

an ichthyological baseline for this poorly known region.

\section{MATERIALS AND METHODS}

\section{Description of study site}

The small fishing village of Andavadoaka $\left(22^{\circ} 07 \mathrm{~S}\right.$, $43^{\circ} 23 \mathrm{E}$, Fig. 1), situated at the extreme northern end of this reef system, some $140 \mathrm{~km}$ north of the Grand Récif, lies on the edge of a shallow lagoon, protected from the open Mozambique Channel by extensive coral reefs. In addition to the fringing reefs that characterise much of the coastal marine environment of the south-west, several banks rise up in the shallow waters on the seaward side of these reefs, and a series of 5 offshore islands, numerous uninhabited islets and submerged barrier reefs also support substantial coral growth.

The Andavadoaka reef system comprises 3 major reef types: inshore fringing reefs; seaward fore-reefs and lagoonal patch reefs. Inshore

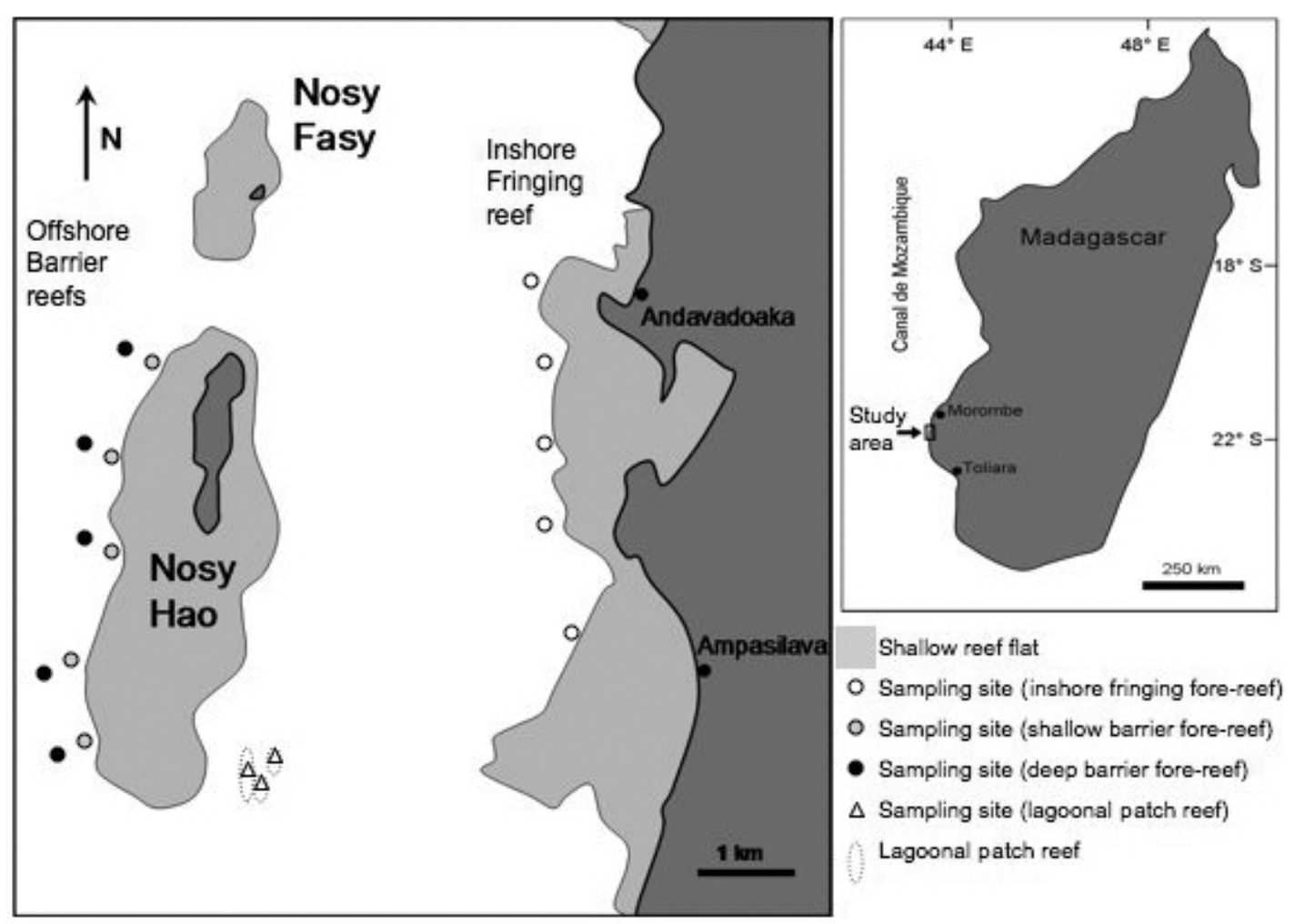

Fig. 1. Location of Andavadoaka and positions of spatial assemblage survey sites fringing reefs are found adjacent to the coast following the length of much of the coastline in the region, descending to, and terminating at, the lagoonal floor at approximately $10 \mathrm{~m}$ depth. These reefs mark the start of a wide lagoon, between 10 and $30 \mathrm{~m}$ in depth, which runs to approximately 8 $\mathrm{km}$ offshore before shelving more steeply towards the continental shelf break. Between 6 and $8 \mathrm{~km}$ offshore, a broken line of islands and sand cays, each fringed on the seaward side by a barrier fore-reef, protects much of the shallow lagoonal environment from open ocean swells. Within the lagoon itself numerous small patch reefs can be found between 10 and $30 \mathrm{~m}$ depth (Figure 1 ).

\section{Methodology}

In order to establish baseline data to compile a regional inventory of fish species present in the region, ongoing sightings of all species were recorded by the authors from all day-time research dives undertaken for a period of one year at reefs throughout the Andavadoaka region, between April 2004 and April 2005.

Alongside this total species census, surveys of spatial assemblages of fish populations wer undertaken at a number of selected sites within the region's reef systems, in order to ascertain relative species abundance between different reef types within the region. These studies were carried out through the collection of site-specific fish specie assemblage data during daytime dives at the end of the austral summer (February-March 2005) over a 5-week period.

For this site-specific study, inshore fringing fore-reefs were surveyed at a depth of between 5 an $10 \mathrm{~m}$. Patch reefs were surveyed at depths between 10 and $18 \mathrm{~m}$, and barrier fore-reefs were surveyed in two depth zones; $5-10 \mathrm{~m}$ and $10-18 \mathrm{~m}$. Surveys of all sites were performed by a visual census technique using a single observer to minimise observer bias, according to the visual census methodology of English et al. (1997), using 30 minute timed swims, with careful recording of each species observed on an underwater slate. Species unidentifiable in situ were recorded with a camera for subsequent expert identification. Estimates of numbers of individual of each species were made using a semi-quantitative root 4 scale to facilitate recording of large numbers of fish, as shown in Table 1.

Visual censuses typically underestimate cryptic species (Fowler, 1987). Moreover, it is not possible to standardise the survey effort spent in actively 'hunting' for cryptic species that might be camouflaged on the benthos or concealed in reef crevices. Therefore to minimise observer bias, only non-cryptic species were recorded for the site-specific spatial assemblage study: species that required active searching on the reef were not included. For the purpose of this study, non-cryptic fish species were considered as all fish species not showing cryptic patterning or behaviour, and being large enough to be clearly and immediately visible to surveyors, thus not skewing results of surveys. In excluding cryptic species it was assumed that the proportion of cryptic species at a reef site remain approximately constant between different sites and depths of coral reefs. Based on this assumption, this preclusion would not affect measures of the relative species abundance between sites.

Each selected geomorphological class or reef (inshore fringing fore-reefs; patch reefs; shallow barrier fore-reefs; and deep barrier fore-reefs was surveyed at five randomly selected survey sites, with the exception of the patch reefs, which were surveyed three times (Figure 1). Efforts were made to ensure that different survey sites within each geomorphological class of reef showed a similar reefscape (Gonzalez-Gandara et al. 1999) to the other survey sites carried out within that geomorphological reef class. In addition, the time of surveying was standardised between 0900 and 1400 to avoid the possible effect of temporal variation in assemblages. To minimise the effect of disturbance caused by divers entering the water, prior to each survey divers waited for 5 minutes to allow the fish in the area to return to normal behaviour.

\section{Data Analysis}

In addition to basic descriptive analysis of data, multivariate analyses were carried out to test for differences between samples. It was recognised that similarities between samples calculated on original species abundance values were likely to be dominated by a small number of highly abundant species. The root 4 transformation $(\sqrt[4]{ } \sqrt{y})$, 
Table 1: Inventory of the reef fishes of the Andavadoaka region (depths 0-30m). Species in bold observed during spatial assemblage surve

\begin{tabular}{|c|c|c|c|}
\hline Species & g Habit & Species & Feeding Habit \\
\hline \multicolumn{2}{|l|}{ CARCHARINIDAE } & \multicolumn{2}{|l|}{ Synanceia verrucosa Bloch \& Schneider, } \\
\hline $\begin{array}{l}\text { Carcharhinus falciformis (Müller \& } \\
\text { Henle, 1839) }\end{array}$ & & & 6 \\
\hline $\begin{array}{l}\text { Henle, 1839) } \\
\text { Triaenodon obesus (Rüppell, 1837) }\end{array}$ & $\begin{array}{l}6 \\
4\end{array}$ & SERRANIDAE & \\
\hline SPHYRNIDAE & & $\begin{array}{l}\text { Aethaloperca rogaa (Forsskål, 1775) } \\
\text { Anyperodon leucogrammicus }\end{array}$ & 5 \\
\hline Sphyrna lewini (Griffith \& Smith, 1834) & 6 & $\begin{array}{l}\text { (Valenciennes, 1828) } \\
\text { Cephalopholis argus Bloch \& Schneider, }\end{array}$ & 6 \\
\hline DASYATIDAE & & 1801 & 6 \\
\hline Taeniura lymma (Forsskål, 1775) & 5 & $\begin{array}{l}\text { Cephalopholis miniata (Forsskål, 1775) } \\
\text { Cephalopholis urodeta (Forster, 1801) }\end{array}$ & $\begin{array}{l}5 \\
5\end{array}$ \\
\hline MOBULIDAE & & Epinephelus caeruleopunctatus (Bloch, & \\
\hline Manta birostris (Walbaum, 1792) & 7 & 1790) & 6 \\
\hline Mobula japenica (Müller \& Henle, 1841) & 7 & $\begin{array}{l}\text { Epinephelus fasciatus (Forsskål, 1775) } \\
\text { Epinephelus flavocaeruleus (Lacepède, }\end{array}$ & 5 \\
\hline MURAENIDAE & & 1801) & 6 \\
\hline Echidna nebulosa (Ahl, 1789) & 5 & Epinephelus hexagonatus (Forster in & \\
\hline Gymnomuraena zebra (Shaw, 1797) & 5 & Bloch \& Schneider, 1801) & 5 \\
\hline Gymnothorax favagineus Bloch \& & & Epinephelus lanceolatus (Bloch, 1790) & 5 \\
\hline Schneider, 1801 & 5 & Epinephelus malabaricus (Bloch \& & \\
\hline Gymnothorax flavimarginatus (Rüppell, 1830) & 6 & Schneider, 1801) & 5 \\
\hline Gymnothorax javanicus (Bleeker, 1859) & 5 & Epinephelus merra Bloch, 1793 & 6 \\
\hline Gymnothorax meleagris (Shaw, 1795) & 4 & Epinephelus ongus Bloch, 1790 & 5 \\
\hline Gymnothorax undulatus (Lacepède, 1803) & 5 & Epinephelus rivulatus (Valenciennes, 1830) & 6 \\
\hline Pseudechidna sp (Bleeker, 1859) & 5 & Epinephelus spilotoceps Schultz, 1953 & 5 \\
\hline Rhinomuraena quaesita Garman, 1888 & 6 & Epinephelus tukula Morgans, 1959 & 5 \\
\hline Siderea picta (Ahl, 1789) & 5 & $\begin{array}{l}\text { Nemanthias carberryi Smith, } 1954 \\
\text { Plectropomus laevis (Lacenède, 1801) }\end{array}$ & $\begin{array}{l}7 \\
5\end{array}$ \\
\hline SYNODONTIDAE & & Plectropomus punctatus Quoy \& Gaimard, & \\
\hline Saurida gracilis (Quoy \& Gaimard, 1824) & 6 & 1824 & 6 \\
\hline Synodus dermatogenys Fowler, 1912 & 6 & $\begin{array}{l}\text { Pseudanthias squamipinnis Peters, } 1855 \\
\text { Variola albimarginata Baissac, } 1953\end{array}$ & $\begin{array}{l}7 \\
6\end{array}$ \\
\hline HOLOCENTRIDAE & & Variola louti (Forsskål, 1775) & 6 \\
\hline Myripristis adusta Bleeker, 1853 & 8 & & \\
\hline Myripristis berndti Jordan \& Evermann, 1903 & 5 & APOGONIDAE & \\
\hline Myripristis $s p$ Cuvier, 1829 & 5 & Apogon aureus (Lacepède, 1802) & 4 \\
\hline Myripristis kuntee Valenciennes, 1831 & 8 & Apogon cookii Macleay, 1881 & 4 \\
\hline Myripristis murdjan Forsskål, 1775 & 8 & Apogon cyanosoma Bleeker, 1853 & 5 \\
\hline Neoniphon sammara Forsskål, 1775 & 5 & Apogon fraenatus Valenciennes, 1832 & 5 \\
\hline Sargocentron caudimaculatum Rüppell, 1838 & 5 & Archamia fucata (Cantor, 1849) & 4 \\
\hline Sargocentron diadema Lacepède, 1802 & 5 & Cheilodipterus macrodon (Lacepède, 1802) & 8 \\
\hline Sargocentron melanospilos Bleeker, 1858 & 5 & Cheilodipterus quinquelineatus Cuvier, & \\
\hline Sargocentron spiniferum Forsskål, 1775 & 5 & 1828 & 5 \\
\hline AULOSTOMIDAE & & PRIACANTHIDAE & \\
\hline $\begin{array}{l}\text { Aulostomus chinensis (Linnaeus, 1766) } \\
\text { FISTULARIDAE }\end{array}$ & 6 & Priacanthus hamrur (Forsskål, 1775) & 5 \\
\hline Fistularia commersonii Rüppell, 1838 & 4 & $\begin{array}{l}\text { GERREIDAE } \\
\text { Gerres oyena (Forsskål, 1775) }\end{array}$ & 4 \\
\hline SCORPAENIDAE & & & \\
\hline Pterois antennata (Bloch, 1787) & 4 & HAEMULIDAE & \\
\hline Pterois sp Cuvier, 1829 & 4 & Diagramma pictum (Thunberg, 1792) & 4 \\
\hline Pterois miles (Bennett 1825) & 6 & Plectorhinchus chubbi (Regan, 1919) & 5 \\
\hline Scorpaenopsis sp Randall \& Eschmeyer, 2001 & 6 & Plectorhinchus flavomaculatus (Cuvier, & \\
\hline Scorpaenopsis diabolus (Cuvier, 1829) & 6 & 1830) & 5 \\
\hline
\end{tabular}

\section{Contd. from page 4}

\section{Species}

Feeding Habit

Plectorhinchus gaterinus (Forsskål, 1775)

Plectorhinchus orientalis (Bloch, 1793)

Plectorhinchus picus (Cuvier, 1830)

Plectorhinchus plagiodesmus Fowler 1935

Plectorhinchus playfairi (Pellegrin, 1914)

Plectorhinchus sordidus (Klunzinger, 1870)

\section{LUTJANIDAE}

Aprion virescens Valenciennes, 1830 Lutjanus argentimaculatus (Forsskål, 1775) Lutjanus bohar (Forsskăl, 1775) Lutjanus fulvifatamma (Forsskål, 1775) Lutjanus fulvus (Forster in Bloch \& Scheider, 801)

Lutjanus gibbus (Forsskål, 1775)

Lutjanus kasmira (Forsskål, 1775)

Lutjanus lutjanus Bloch, 1790

Lutjanus monostigma (Cuvier, 1828)

Lutjanus notatus (Cuvier, 1828)

Lutjanus sebae (Cuvier, 1816)

CAESIONIDAE

Caesio caerulaurea Lacepède, 180

Caesio lunaris Cuvier, 1830

Caesio striata Rüppell, 1830

Caesio xanthonota Bleeker, 1853

Pterocaesio capricornis Smith \& Smith,

1963

Pterocaesio chrysozona (Cuvier, 1828)

Pterocaesio marri (Schultz, 1953)

Pterocaesio tile (Cuvier, 1830)

LETHRINIDAE

Gnathodentex aurolineatus (Lacepède,

1802)

Lethrinus borbonicus Valenciennes, 1830

Lethrinus conchyliatus (Smith, 1959)

Lethrinus harak (Forsskål, 1775)

Lethrinus lentjan (Lacepède, 1802)

Lethrinus microdon Valenciennes, 1830

Lethrinus nebulosus (Forsskả, 1775)

Lethrinus obsoletus (Forsska, 1775)

Lethrinus olivaceus Valenciennes, 1830

Monotaxis grandoculis (Forsth1, 1775)

MULLIDAE

Mulloidichthys flavolineatus (Lacepède,

Mulloidich

Mulloidichthys vanicolensis

(Valenciennes, 1831)

Parupeneus barberinus (Lacepède, 1801)

Parupeneus trifasciatus (Lacepède, 1801)

Parupeneus cyclostomus (Laceède, 1801)

Parupeneus indicus (Shaw, 1803)

Parupeneus macronema (Lacepède, 1801)

Parupeneus pleurostigma (Bennett, 1832)
Species

Feeding Habi

Parupeneus rubescens (Lacepède, 1801)

KYPHOSIDAE

Kyphosus cinerascens Forsskål, 1775

EPHIPPIDAE

Platax orbicularis (Forsskål, 1775)

POMACANTHIDAE

Apolemichthys trimaculatus (Lacepède

1831)

Centropyge acanthops (Norman, 1922) Centropyge bispinosus (Günther, 1860) Centropyge multispinis (Playfair, 1867) Pomacanthus chrysurus (Cuvier, 1831) Pomacanthus imperator (Bloch, 1787) Pomacanthus

Pygoplites diacanthus (Boddaert, 1772)

CHAETODONTIDAE

\section{Chatodon auriga Forsskål, 177}

Chaetodon bennetti Cuvier in Cuvier \&

Vlenciennes, 183

.

Bennett, 1832

Chaetodon unimaculatus interruptus Ahl,

Chaetodon kleinii Bloch, 1790

Chaetodon lineolatus (Quoy \& Gaimard,

1831 in Cuvier \& Valenciennes)

Chaetodon lunula (Lacepède, 1802)

Chaetodon melannotus (Bloch \&

Schneider, 1801)

Chaetodon meyeri Bloch \& Schneider, 1801 Chaetodon trifascialis Quoy \& Gaimard,

1825

Chaetodon trifasciatus Mungo Park, 1797

Chaetodon vagabundus Linnaeus, 1758

Chaetodon xanthocephalus Bennett, 1832

Chaetodon zanzibariensis Playfair, in

Playfair \& Günther, 1867

orcipiger longirostris (Broussonet, 1782)

(Bemitonus acuminatus (Linet, 1831)

Heichus momaces (Cuvi 1831)

ECHENEIDA

Echeneis naucrates Linnaeus, 1758

CARANGIDAE

Carangoides ferdau (Forsskål, 1775)

Carangoides fulvoguttatus (Forsskål, 1775)

Caranx ignobilis (Forsskål, 1775)
1 
Contd. from page 5

\begin{tabular}{|c|c|c|c|}
\hline Species & Feeding Habit & Species & 'eeding Habit \\
\hline Caranx melampygus Cuvier \& & & Pomacentrus trichrourus Günther, 1877 & 2 \\
\hline Valenciennes, 1833 & 4 & Pomacentrus trilineatus Cuvier, 1830 & 2 \\
\hline Caranx heberi (Bennet 1830) & 4 & Stegastes fasciolatus (Ogilby, 1889) & 1 \\
\hline $\begin{array}{l}\text { Elagatis bipinnulata (Quoy \& Gaimard, } \\
1825 \text { ) }\end{array}$ & 4 & LABRIDAE & \\
\hline Gnathanodon speciosus (Forsskål, 1775) & 4 & $\begin{array}{l}\text { Anampses caeruleopunctatus Rüppell, } 1829 \\
\text { Anampses meleagrides Valenciennes, } 1840\end{array}$ & $\begin{array}{l}4 \\
4\end{array}$ \\
\hline CIRRHITIDAE & & Anampses twistii Bleeker, 1856 & 4 \\
\hline Cirrhitichthys oxycephalus (Bleeker, 1855) & 4 & Bodianus anthioides Bennett, 1832 & 4 \\
\hline Cirrhitus pinnulatus (Forster, 1801) & 4 & Bodianus axillaris Bennett, 1832 & 4 \\
\hline Paracirrhites arcatus Cuvier in Cuvier \& & & Bodianus bilunulatus bilunulatus & \\
\hline Valenciennes, 1829 & 4 & (Lacepède), 1801 & 4 \\
\hline Paracirrhites forsteri (Bloch \& Schneider, & & Bodianus diana Lacepède, 1801 & 4 \\
\hline 1801) & 4 & $\begin{array}{l}\text { Cheilinus chlorourus Bloch, } 1791 \\
\text { Cheilinus fasciatus fasciatus Bloch, } 1791\end{array}$ & $\begin{array}{l}4 \\
4\end{array}$ \\
\hline PEMPHERIDAE & & Cheilinus trilobatus Lacépède, 1801 & 4 \\
\hline Parapriacanthus ransonneti Steindachner, & & Cheilinus undulatus Rüppell, 1835 & 4 \\
\hline 1870 & 8 & Cheilio inermis Forsskål, 1775 & 4 \\
\hline Pempheris oualensis Cuvier, 1831 & 8 & Coris aygula Lacepède, 1801 & 4 \\
\hline Pempheris schwenkii Bleeker, 1855 & 8 & Coris caudimacula Quoy \& Gaimard, 1834 & 4 \\
\hline Pempheris vanicolensis Cuvier, 1831 & 8 & $\begin{array}{l}\text { Epibulus insidiator Pallas, } 1770 \\
\text { Gomphosus caeruleus caeruleus Lacepède, }\end{array}$ & 4 \\
\hline POMACENTRIDAE & & 1801 & 4 \\
\hline Abudefduf natalensis Hensley \& Randall, & & Halichoeres hortulanus Lacepède, 1801 & 4 \\
\hline $1983^{\circ}$ & 2 & Halichoeres scapularis Bennett, 1832 & 4 \\
\hline Abudefduf notatus (Day, 1870) & 2 & Halichoeres sp, & 4 \\
\hline Abudefduf septemfasciatus (Cuvier, 1830) & 2 & Hemigymnus fasciatus Bloch, 1792 & 4 \\
\hline Abudefduf sexfasciatus (Lacepède, 1801) & 2 & Hemigymnus melapterus Bloch, 1791 & 4 \\
\hline Abudefduf sordidus (Forsksål, 1775) & 2 & Hologymnosus annulatus Lacepède, 1801 & 4 \\
\hline Abudefduf sparoides (Quoy \& Gaimard, & & Hologymnosus doliatus Lacepède, 1801 & 4 \\
\hline 1825) & 2 & Labroides bicolor Fowler \& Bean, 1928 & 4 \\
\hline Abudefduf vaigiensis (Quoy \& Gaimard, & & Labroides dimidiatus Valenciennes, 1839 & 4 \\
\hline 1825) & 2 & Macropharyngodon bipartitus Smith, 1957 & 4 \\
\hline Amblyglyphidodon indicus Allen \& & & Novaculichthys macrolepidotus Bloch, 1791 & 4 \\
\hline Randall, 2002 & 2 & Oxycheilinus diagrammus Lacepède, 1801 & 4 \\
\hline Amphiprion akallopisos Bleeker, 1853 & 2 & Stethojulis albovittata Bonnaterre, 1788 & 4 \\
\hline Amphiprion latifasciatus Allen, 1972 & 2 & Thalassoma amblycephalum Bleeker, 1856 & 4 \\
\hline Chromis dimidiata (Klunzinger, 1871) & 7 & Thalassoma hardwicke Bennett, 1830 & 4 \\
\hline Chromis lepidolepis Bleeker, 1877 & 7 & Thalassoma hebraicum Lacepède, 1801 & 4 \\
\hline Chromis leucura Gilbert, 1905 & 2 & Thalassoma lunare Linnaeus, 1758 & 4 \\
\hline Chromis nigrura Smith, 1960 & 7 & Thalassoma trilobatum Lacepède, 1801 & 4 \\
\hline Chromis ternatensis (Bleeker, 1856) & 7 & Wetmorella albofasciata Schultz \& & \\
\hline Chromis viridis Cuvier, 1830 & 2 & Marshall, $1954^{\circ}$ & 4 \\
\hline Chromis weberi Fowler \& Bean, 1928 & 7 & Xyrichtys pavo Valenciennes, 1840 & 4 \\
\hline Chrysiptera annulata (Peters, 1855) & 2 & & \\
\hline Chrysiptera unimaculata (Cuvier, 1830) & 2 & SCARIDAE & \\
\hline Dascyllus aruanus (Linnaeus, 1758) & 7 & Calotomus carolinus (Valenciennes, 1840) & 1 \\
\hline Dascyllus carneus Fischer, 1885 & 2 & Cetoscarus bicolor (Rüppell, 1829) & 1 \\
\hline Dascyllus trimaculatus (Rüppell, 1829) & 7 & Chlorurus cyaneascens (Valenciennes, & \\
\hline Neoglyphidodon melas (Cuvier, 1830) & 4 & 1840) & 1 \\
\hline Plectroglyphidodon dickiï (Liénard, 1839) & 2 & Chlorurus sordidus (Forsskål, 1775) & 1 \\
\hline Plectroglyphidodon lacrymatus (Quoy & & Chlorurus strongylocephalus (Bleeker, 1854) & 1 \\
\hline \&Gaimard, 1825) & 2 & Hipposcarus harid (Forsskål, 1775) & 1 \\
\hline Pomacentrus baenschi Allen, 1991 & 2 & Scarus caudofasciatus (Günther, 1862) & 1 \\
\hline Pomacentrus caeruleus Quoy \& & & Scarus festivus Valenciennes in Cuvier & \\
\hline Gaimard, 1825 & 2 & \& Valenciennes, 1840 & 1 \\
\hline Pomacentrus sulfureus Klunzinger, 1871 & 2 & Scarus frenatus Lacepède, 1802 & 1 \\
\hline
\end{tabular}

Contd. from page 6

\begin{tabular}{|c|c|}
\hline Species & eeding Habit \\
\hline Scarus ghobban Forsskål, 1775 & 1 \\
\hline Scarus globiceps Valenciennes, 1840 & 1 \\
\hline Scarus niger Forsskål, 1775 & 1 \\
\hline Scarus rubroviolaceus Bleeker, 1847 & 1 \\
\hline Scarus russelli Valenciennes, 1840 & 1 \\
\hline \multicolumn{2}{|l|}{ Scarus scaber Valenciennes in Cuvier \& } \\
\hline Valenciennes, 1840 & 1 \\
\hline Scarus tricolor Bleeker, 1847 & 1 \\
\hline \multicolumn{2}{|l|}{ SPHYRAENIDAE } \\
\hline Sphyraena barracuda (Walbaum, 1792) & 4 \\
\hline Sphyraena flavicauda Rüppell, 1838 & 4 \\
\hline Sphyraena sp Cuvier, 1829 & 6 \\
\hline \multicolumn{2}{|l|}{ PINGUIPEDIDAE } \\
\hline Parapercis hexophtalma (Cuvier, 1829) & 4 \\
\hline \multicolumn{2}{|l|}{ BLENNIDAE } \\
\hline Ecsenius nalolo Smith, 1959 & 1 \\
\hline Meiacanthus mossambicus Smith, 1959 & 1 \\
\hline Plagiotremus rhinorhynchos (Bleeker, 1852) & 8 \\
\hline Plagiotremus tapeinosoma (Bleeker, 1857) & 2 \\
\hline \multicolumn{2}{|l|}{ MICRODESMIDAE } \\
\hline Gunnelichthys sp Dawson, 1968 & 7 \\
\hline Nemateleotris magnifica Fowler, 1938 & 7 \\
\hline Ptereleotris evides (Jordan \& Hubbs, 1925) & 7 \\
\hline \multicolumn{2}{|l|}{ GOBIIDAE } \\
\hline Amblyeleotris steinitzi (Klausewitz, 1974) & 4 \\
\hline Amblygobius tekomaji (Whitley, 1940) & 4 \\
\hline \multirow{2}{*}{\multicolumn{2}{|c|}{ Cryptocentrus strigilliceps (Jordan \& Seale, }} \\
\hline & 4 \\
\hline Gobiodon citrinus (Rüppell, 1838) & 4 \\
\hline Istigobius decoratus (Herre, 1927) & 4 \\
\hline Oplopomus oplopoтиs Valenciennes, 1837 & 4 \\
\hline Valenciennea strigata (Broussonet, 1782) & 4 \\
\hline \multicolumn{2}{|l|}{ ACANTHURIDAE } \\
\hline Acanthurus dussumieri Valenciennes, 1835 & 1 \\
\hline Acanthurus leucosternon Bennet, 1833 & 1 \\
\hline Acanthurus lineatus (Linnaeus, 1758) & 1 \\
\hline Acanthurus mataRussell in Cuvier, 1829 & 7 \\
\hline \multicolumn{2}{|l|}{ Acanthurus nigricauda Duncker \& Mohr, } \\
\hline 1929 & 1 \\
\hline Acanthurus nigrofuscus (Forsskål, 1775) & 1 \\
\hline Acanthurus tennenti Günther, 1861 & 1 \\
\hline Acanthurus thompsoni Fowler, 1923 & 1 \\
\hline \multicolumn{2}{|l|}{ Acanthurus triostegus triostegus } \\
\hline (Linnaeus, 1758) & 1 \\
\hline Ctenochaetus binotatus Randall, 1955 & 1 \\
\hline \multicolumn{2}{|l|}{ Ctenochaetus striatus (Quoy \& Gaimard, } \\
\hline 1825) & 1 \\
\hline Ctenochaetus strigosus (Bennet, 1828) & 1 \\
\hline Naso annulatus (Quoy \& Gaimard, 1825) & 1 \\
\hline Naso brevirostris (Cuvier, 1829) & 1 \\
\hline Naso elegans (Rüppell, 1829) & 1 \\
\hline
\end{tabular}

Species

Feeding Habit

Naso hexacanthus (Bleeker, 1855) Naso unicornis (Forsskål, 1775) Naso vlamingi (Valenciennes, 1835) Zebrasoma velifer (Bloch, 1795) Zebrasoma gemmatum (Valenciennes, 1835)

Zebrasoma scopas (Cuvier, 1829)

ZANCLIDAE

Zanclus canescens (Linnaeus, 1758)

SIGANIDAE

Siganus argenteus (Quoy \& Gaimard,

1825)

Siganus luridus (Rüppell, 1829)

Siganus stellatus (Forsskål, 1775)
Siganus sutor (Valenciennes, 1835)

BOTHIDAE

Bothus pantherhinus (Rüppell, 1830)

SCOMBRIDAE

Gymnosarda unicolor (Rüppell, 1836)

Scomberomorus commerson (Lacepède,

1800)

BALISTIDAE

Balistapus undulatus (Mungo Park, 1797)

Balistoides conspicillum (Bloch \&

Schneider, 1801)

Balistoides viridescens (Bloch \&

Schneider, 1801)

Melichthys niger (Bloch, 1786)

Odonus niger (Rüppell, 1836)

Rhinecanthus aculeatus (Linnaeus, 1758)

Sufflamen bursa (Bloch \& Schneider, 1801)

Sufflamen chrysopterus (Bloch \&

Schneider, 1801)

MONACANTHIDAE

Amanses scopas (Cuvier, 1829)

Cantherhines pardalis (Rüppell, 1837)

Oxymonacanthus longirostris (Bloch \&

Schneider, 1801)

OSTRACIIDAE

Ostracion cubicus (Linnaeus, 1758)

Ostracion meleagris Shaw, 1796

TETRADONTIDAE

Arothron hispidus (Linnaeus, 1758

Arothron immaculatus (Bloch \&

Schneider, 1801)

Arothron meleagris (Lacepède, 1798)

Arothron nigropunctatus Bloch \&

1




\section{Species}

Feeding Habit

\section{Schneider, 1801}

Arothron stellatus (Bloch \& Schneider, 1801) Canthigaster bennetti (Bleeker, 1854) Canthigaster solandri (Richardson, 1845) Canthigaster valentini (Bleeker, 1853) Torquigener flavimaculosus Hardy \& Randall, 1983

DIODONTIDAE

Diodon liturosus Shaw, 1804

BELONIDAE

Strongylura incisa (Valenciennes, 1846)

(Péron \& Lesueur,

1821)

CENTRISCIDAE

Aeoliscus strigatus (Günther, 1860)

DACTYLOPTERIDAE

Dactyloptena orientalis (Cuvier, 1829)

GRAMMISTIDAE

Grammistes sexlineatus Thunberg, 1793

HEMIRAMPHIDAE

Hemiramphus far Forrskål, 1775

NEMIPTERIDAE

Scolopsis bimaculatus Rüppell, 182

Scolopsis ghanam (Forsskำ, 1775)

Scolopsis vosmer (Bloch, 1792)

\section{ORECTOLOBIDAE}

Stegostoma fasciatum (Hermann, 1783)

PLATYCEPHALIDAE

Papilloculiceps longiceps (Cuvier, 1829)

PLOTOSIDAE

Plotosus lineatus (Thunberg, 1787)

PSEUDOCHROMIDAE

Pseudochromis dutoiti Smith, 1955

RHINOBATIDAE

Rhynchobatus djiddensis (Forsskål, 1775)

SPARIDAE

Acanthopagrus bifasciatus (Forsskål, 1775)

SYNGNATHIDAE

Corythoichthys sp Dawson \& Randall, 1975 Doryramphus excisus excisus Kaup, 1856

Feeding Habits- 1: Herbivore, 2: Omnivore, 3: Browser of Sessile Invertebrates, 4: Diurnal Carnivore, 5: Nocturnal Carnivore, 6: Piscivore, 7: Diurnal Planktivore, 8: Nocturnal Planktivore applied when recording data in the field, provided a means of down-weighting the importance of highly abundant species so that sample similarities depended not only on their values but also those of less common ('mid-range') species. Hierarchical agglomerative clustering with group-average linking, based on Bray-Curtis sample similarities (appropriate for delineating groups into distinct community structure) was used to find natural groupings of samples using Primer 6 multivariate analysis software. Sites were then discriminated on the basis of their species composition using non-metric multi-dimensional scaling (MDS) and analysis of similarities (ANOSIM) testing, the latter routine being used to identify significant differences between groups of samples defined a priori. By using the non-metric MDS approach, different reef sites (samples) can be considered to have differing fish community compositions when different samples derived from one reef site form a cluster that is distinct from replicates within other sites.

A theoretical value of species richness $\left(\mathrm{SR}_{\mathrm{th}}\right)$ was calculated, based on the regression formula produced by Allen \& Werner (2002), to account for the effect of sampling effort in results obtained from the global species census, and to facilitate comparative analysis of species richness data with other reference sites in the Indo-Pacific region. Calculation of $\mathrm{SR}_{\mathrm{th}}$ is based on an index of fish diversity (coral reef fish diversity index, CFDI). CFDI is equal to the total number of species observed within the acanthuridae, scaridae, labridae, pomacentridae pomacanthidae and chaetodontidae. The theoretical value $\mathrm{SR}_{\mathrm{th}}=4.234$ (CFDI) - 114.446.

In order to analyse spatial variation in trophic assemblages of species recorded at study sites during the one-year study, species were categorised to one of 8 trophic levels using data from previous studies described in literature (Hiatt \& Strasburg 1960; Hobson 1974; Harmelin-Vivien 1979) and from FishBase (http://www.fishbase.org). The 8 categories of feeding habits were: herbivore; omnivore; browser of sessile invertebrates; diurnal carnivore; nocturnal carnivore; piscivore; diurnal planktivore; and nocturnal planktivore. This approach also enabled subsequent trophic assemblage comparisons with other analyses conducted in the region (Durville et al. 2003 Chabanet \& Durville 2005). Planktivores feeding on fish nekton were classified as planktivores rather than piscivores in order to be consistent with the studies of Harmelin-Vivien (1979) and Hiatt \& Strasburg (1960)

\section{RESULTS}

\section{Species Richness}

During the year-long study, a total of 334 species was observed, representing 58 families (Table 1). Of these, 7 species were cartilaginous (Clas Chondrichthyes), while the remaining 327 species were bony fish (Class Osteichthyes). $48.2 \%$ of the species observed belonged to seven major families: labridae (36 species); pomacentridae (31 species); serranidae (23 families); acanthuridae (22 species); chaetodontidae (18 species); scaridae (16 species); and lutjanidae (12 species). Of the remaining 5 amilies, 23 showed only a single species. During the 5-week period of the spatial assemblage study, 187 species were observed, $56 \%$ of the total number of species recorded in the year-long study.

\section{Trophic Structur}

$76 \%$ of total species observed were carnivores, feeding on fish, invertebrates or zooplankton (Fig. 2). Of these, the largest number of species observed (28\% of the total) were diurnal carnivores, such as the labridae. The nocturnal carnivores (such as lethrinidae and lutjanidae) were the second most highly represented, showing $21 \%$ of the total species observed. Piscivores (serranidae), browsers of sessile invertebrates (chaetodontidae) and diurnal planktivores (caesionidae) are similarly represented, each showing $8 \%$ of total species observed. The final group of carnivores, the nocturnal planktivores (such as apogonidae) are represented by $3 \%$ of the total species observed. Omnivores (such as pomacentridae) and herbivores (mainly acanthuridae and scaridae) each represented $11 \%$ of the total species observed.

\section{Spatial variation in assemblage}

Survey stations showed strong similarity in assemblage within reef types, and distinct dissimilarities in assemblage between different reef types (Figures 3 and 4). MDS ordination plots

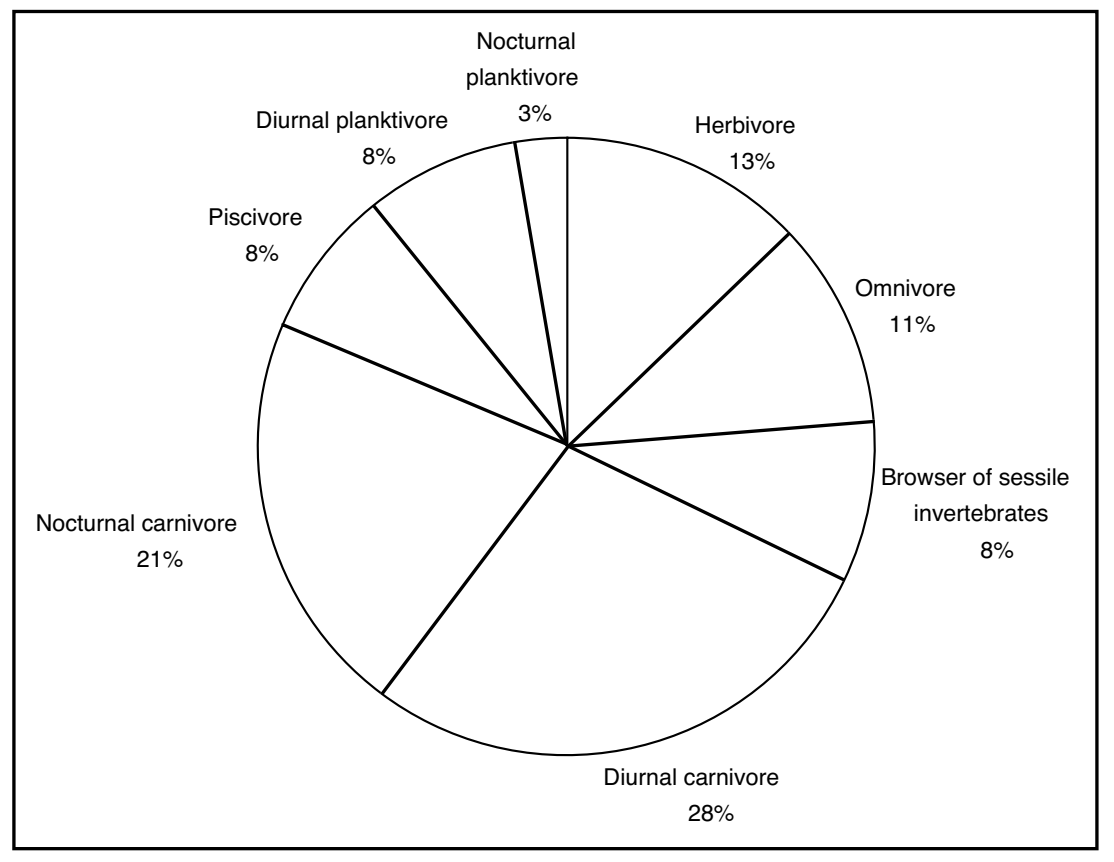

Fig. 2. Trophic guilds of fish species observed in Andavadoaka, expressed as percentage of total species observed during the year-long census study 


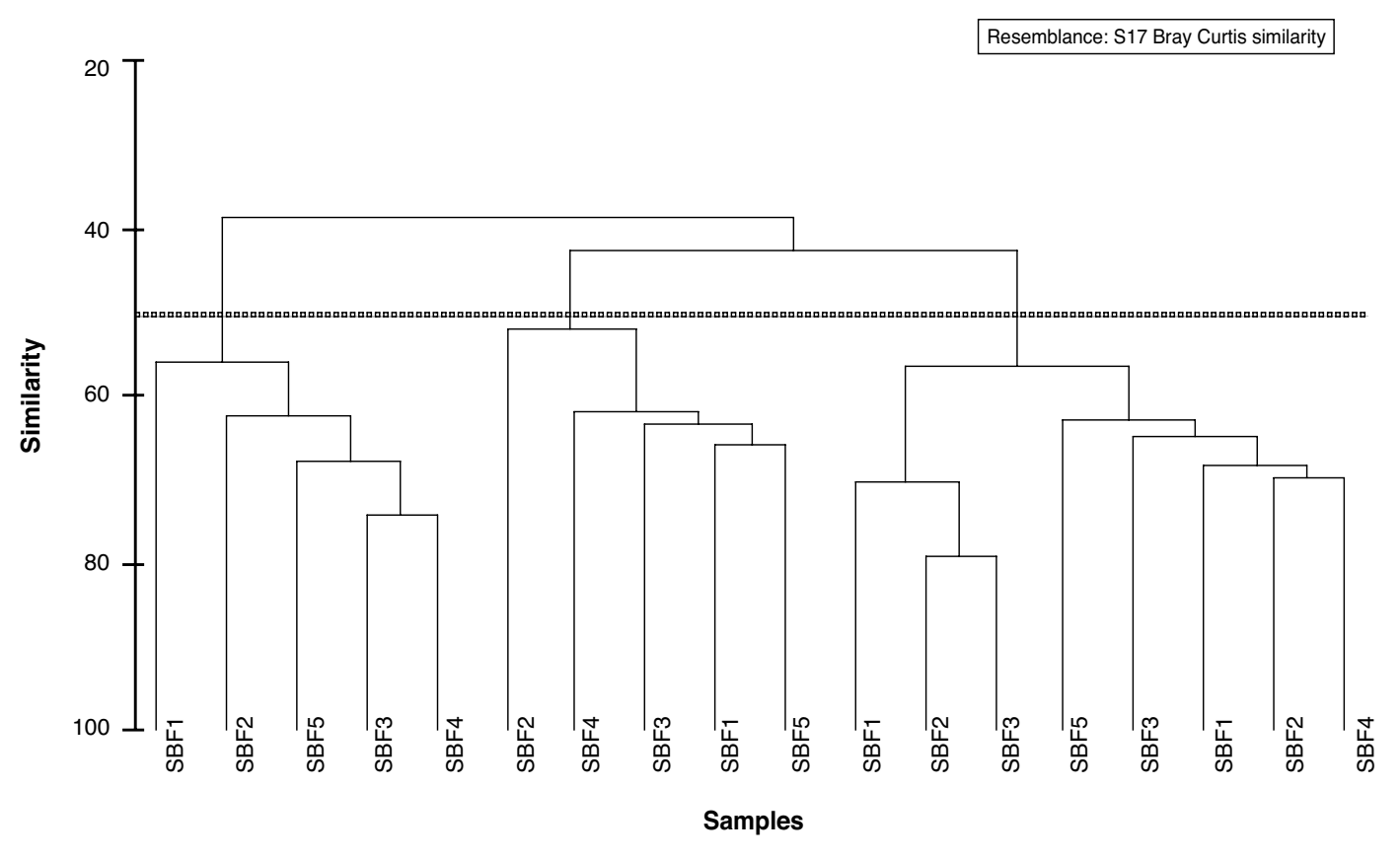

Fig. 3. Hierarchical agglomerative clustering with group-average linking, based on Bray-Curtis sample similarities (appropriate for delineating sample groups by similar community compositions), showing natural groupings of samples
from spatial assemblage study (DBF = deep barrier fore-reef; $\mathrm{SBF}=$ shallow barrier fore-reef, $\mathrm{SFF}=$ shallow fringing fore-reef, DP = deep patch reef)

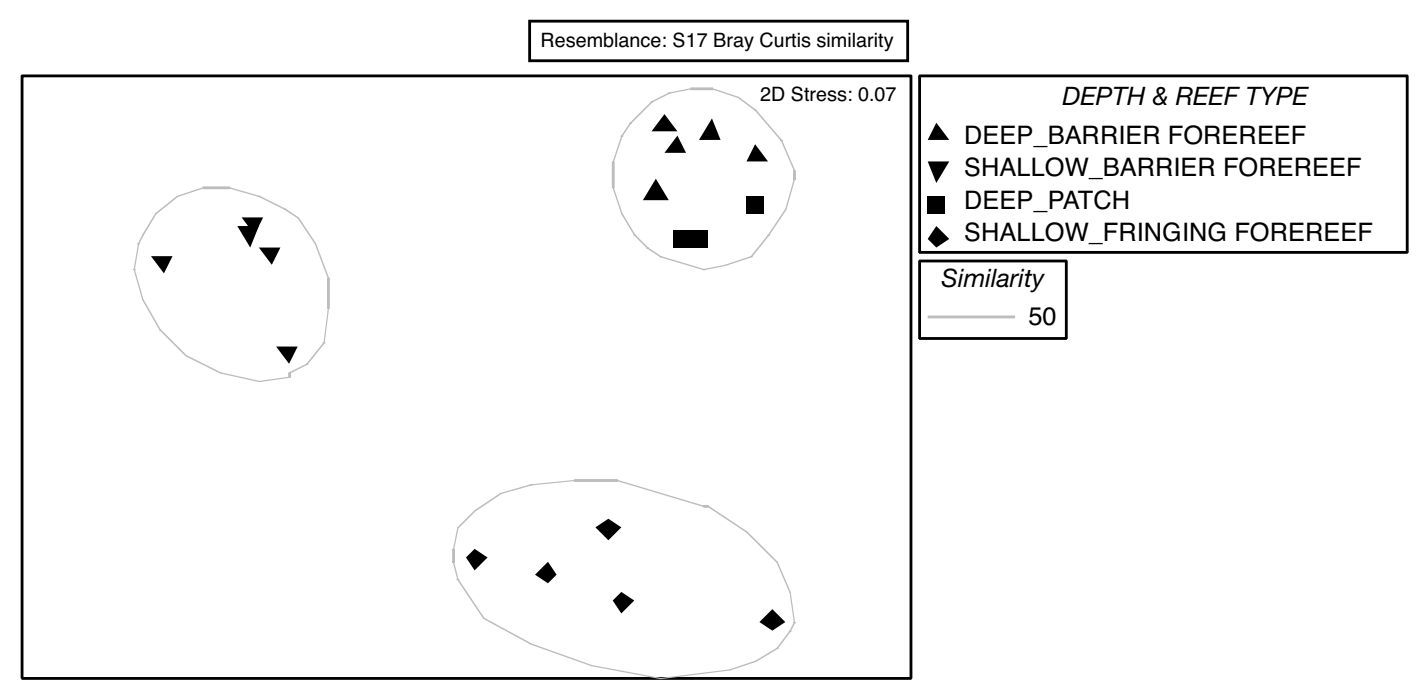

Fig. 4. Non-metric MDS ordination of samples (all depths, all reef types) based on root 4 transformed fish species abundance data showing Bray-Curtis grouping of samples at $50 \%$ resemblance

representing samples as points in 2-dimensional space (such that the relative distances between points represent the rank order of Bray-Curtis dissimilarities of samples) provide a useful means of showing similarities in reef composition between sites. Samples split convincingly between depths sites, which group together and independently of other reef types. Superimposition of Bray-Curtis similarity clusters (Figure 3 ) on this ordination plot emphasises relationships between sample groups.
This observation does not imply that groups have no characteristics in common, but that differen characteristic patterns of fish assemblage are found consistently within the different groups. These differences are confirmed by results of a 2-way crossed ANOSIM test for difference between depths and reef zones across all samples. Results show total separation of samples between depths (global $\mathrm{R}=1.0, \mathrm{p}=0.8 \%$ ) and very strong separation between reef types (global $\mathrm{R}=0.98, \mathrm{p}=$ $0.1 \%)$. In both cases, the observed sample statistic and significance level rejects the null hypotheses that there is no difference in fish species assemblage between different depths and reef types. Fish species assemblages found at deep barrier fore-ree and lagoonal patch reef sites show the strongest between-group similarities (Figure 4): deep barrier fore-reef areas showed an average of 82 species per survey, very similar to the lagoonal patch reefs (average 88 species per survey). All shallow forereef and inshore fringing reef survey sites showed markedly lower numbers of species per survey (59 and 61 respectively).

\section{DISCUSSION}

\section{Species Richness}

A total of 334 species was observed over the year of sampling. As with all visual censuses, underestimation of cryptic species makes it likely that total species numbers are considerably higher than those found in this study (Fowler, 1987). Seasonal variation was observed in certain species, most noticeably in the seasonal arrival of larg shoals of Sphyraena flavicauda in the months of February and March. The majority of reef species (for example Ctenochaetus striatus) were present in surveys throughout the year. This figure of species richness is comparable to that found during other studies in the Western Indian Ocean (Durville $e t a$. 2003; Chabanet 1994; Chabanet 2002; Chabanet \& Durville 2005; Harmelin-Vivien 1979), although some of these studies were performed over much shorter time periods (Durville et al. 2003; Chabanet 2002) with more intensive sampling than was possible during this study (Durville et al 2003). The 187 species observed in the limited sampled surveying during 5 weeks of the spatial assemblage study may represent a temporal "snapshot" of species present at the specific reef zones studied. The species richness for Andavadoaka is higher than that found for the Glorieuses Islands (332 species; Durville et al. 2003), Juan de Nova (299 species; Chabanet \& Durville 2005), Geyser and Zéléé (294 species; Chabanet 1994), Réunion (257 species; Chabanet, 1994) and Mayotte (239 species Chabanet 2002). These results are also consistent with the usual trend of isolated island-associated reefs to show lower diversity than equivalent areas associated with larger land masses (Randall, 1998). According to the CFDI predictor formula an approximate total of 453 species could be expected, indicating that $73 \%$ of the predicted total fish fauna was surveyed. This difference is proportionately similar to published theoretical $\left(\mathrm{SR}_{\mathrm{th}}\right)$ versus observed $\left(\mathrm{SR}_{\mathrm{obs}}\right)$ species richness values for other sites in the region (Chabanet \& Durville 2005).

Despite their comparatively higher species richness, Andavadoaka's reefs are subject to chronic and rapidly growing subsistence and commercial fishing pressures which are not experienced at less exploited reference sites in the Western Indian Ocean (notably Glorieuses Islands, Juan de Nova, and Geyser and Zéléé). Fishing is the primary economic activity for $71 \%$ of Andavadoaka's population of 1200 (Blue Ventures Conservation unpublished data), and communities throughout the region fish for subsistence as well as for income, selling catches to a rapidly growing commercial export market. The population of the Toliara grew by $324 \%$ between 1975 and 1993 (Cooke et al. 2000) and limited employment opportunities and low agricultural productivity resulted in a fivefold increase in the region's fishing population in a period of 17 years leading up until the early 1990s (McVean et al., 2005). Andavadoaka has seen a doubling of population input rate (births and immigration arrivals per year) in the 10 years leading up to 2003, with over $50 \%$ of the population being 14 or under (Blue Ventures Conservation unpublished data).

Alongside direct fishing pressure, the reefs of south-west Madagascar have experienced widespread degradation as a result of the cora bleaching and mortality events of 1998 and 2001 (Cooke, 2003). Almost all shallow reef areas in 
the region are now highly degraded. Low levels of hard coral and high algal dominance in these areas have resulted in a structurally poor environment, particularly in shallow reef environments; a factor which has been shown to lead to lower fish species richness in other areas (Gratwicke \& Speight, 2005).

The adverse effects of bleaching-associated coral mortality and fishing on coral reef fish species richness are well documented (McClanahan 1994; Wantiez et al. 1997; Jones et al. 2004). It is likely that Andavadoaka's fish species richness has been reduced by these impacts. Supporting this assertion, the two areas of shallow reef studied showed lower species numbers than the deeper areas. These shallow sites experience greater fishing pressures than deeper reefs, and are likely to have experienced greater thermal stress than deeper sites during bleaching events.

Andavadoaka's species richness is far higher than that found on the Grand Récif of Toliara in 1979 by Harmelin-Vivien, despite experiencing widespread reef mortality which was not present at the time of the 1979 study. Fishing pressures were also unlikely to have been at existing levels of intensity during the 1979 Toliara study, which is currently the only comparable fish biodiversity assessment for southern Madagascar. The higher species richness recorded in Andavadoaka, despite their current degraded status, suggests that the area is of significant regional importance for fish biodiversity and conservation.

\section{Trophic Structure}

Despite the degraded state of Andavadoaka's shallower reefs, which could be expected to impact the number of carnivorous species observed on the reef (Harmelin-Vivien 1992), 74\% of species

Table 2. $\log 4$ abundance scale

\begin{tabular}{cc}
\hline Root 4 value & Abundance \\
\hline $0:$ & 0 \\
$1:$ & $1-4$ \\
$2:$ & $5-16$ \\
$3:$ & $17-64$ \\
$4:$ & $65-256$ \\
$5:$ & $257+$ \\
\hline
\end{tabular}

observed were carnivores. According to HarmelinVivien (1979), observed carnivore levels on a healthy reef are usually between 60 and $80 \%$, depending on geographic location. This suggests that the fish communities of Andavadoaka are in good health, despite the high fishing effort and mortality episodes that have occurred over the last decade. This indication is similar to that of Durville et al. (2003), who also found very little impact on fish populations following a bleaching event; although in this case the reefs of the Glorieuses islands are dominated by calcareous algae rather than hard corals, and fish populations may have been less susceptible to the effects of reef bleaching than at Andavadoaka reefs.

Notwithstanding these observations of encouraging species richness in Andavadoaka, the longer-term impacts of past mortality events on fish trophic structure and species richness remain to be seen. If Andavadoaka's degraded reefs remain in an algal-dominated state they are likely to suffer further physical deterioration, erosion and decreasing structural complexity, leading to loss of species within key functional groups of reef fish, and substantial reductions of species richness, as has been recorded elsewhere in the south-west Indian Ocean (Graham et al. 2006).

Durville et al. (2003) have compared the levels of carnivores/omnivores/herbivores across the western Indian Ocean area, to which the findings of the current study have been added (see Table 3). These data support the assertion of Durville $e t$ $a l$. that the reefs of the Glorieuses islands and also other reefs of the Mozambique Channel (Geyser, Mayotte, Juan de Nova) come closest to the reefs of south-west Madagascar in terms of reef fish trophic structure.

\section{Spatial variation in assemblage}

With the exception of the deep barrier fore-reef and patch reef sites, there are distinct differences in assemblage between reef types, with pronounced differences between the two shallow reef types as well as between shallow and deep sites. It is well established that fish assemblages change along a depth gradient (Harmelin-Vivien, 1977; McGehee, 1994; Williams, 1991), however within this study the additional large difference between
Table 3. Trophic structure of the fish communities, expressed in percentage of total number of species on different Indo-Pacific coral reefs

\begin{tabular}{llccc}
\hline & & Carnivores & Omnivores & Herbivores \\
\hline Tuléar (Madagascar) & Harmelin-Vivien, 1979 & 74 & 13.5 & 12.5 \\
Réunion & Chabanet, 1994 & 51 & 24 & 25 \\
Mayotte & Chabanet, 2002 & 69 & 12.5 & 18.5 \\
Geyser \& Zélée & Chabanet et al., 2002 & 69 & 16 & 15 \\
Glorieuses & Durville et al., 2003 & 73 & 12 & 15 \\
Juan de Nova & Chabanet \& Durville, 2005 & 73 & 16 & 11 \\
Andavadoaka (Madagascar) & This study & 76 & 11 & 13 \\
\hline
\end{tabular}

the two shallow study areas (barrier fore-reef and inshore fringing fore-reef) indicates that there are contributing factors other than depth alone. Both sites are considered to have suffered extensive degradation from the bleaching events of 1998 and 2001 (Cooke, 2002), however the proximity of the inshore fringing fore-reef to the coast, and its easy accessibility to fishers, means that fishing pressure is much higher at this shallow near-shore ree system. The deep-water reef-fishing techniques of the indigenous Vezo population (currently limited to line fishing) play a role in limiting over-exploitation of the deeper reefs, and it is possible that population of reproductive adults at deeper, less exploited, reef sites may support continued recruitment at more degraded sites in the area, thus maintaining species richness of the shallower sites. In addition to experiencing lower biomass removal through fishing, the shallow barrier fore-reef is a much higher-energy system with greater architectural complexity, exhibiting pronounced spur and groove formations. This habitat is constantly exposed to the prevailing sea surge, and as such is unsuitable for the establishment of territorial benthic herbivore such as pomacentridae. Conversely, the inshore fringing reef is a much lower energy environment, sheltered by the barrier islands and wide lagoon, and capable of supporting higher populations of benthic territorial grazers. As evidence for this suggestion, shallow fore-reef areas showed highe numbers of non-territorial herbivorous species, such as acanthuridae and scaridae, and fewer benthic territorial herbivores.

\section{CONCLUSION}

This baseline study indicates that, despite broad-scale loss of reef structure due to bleaching-related coral mortality, reef fish populations in Andavadoaka are similar both in terms of species richness and trophic structure to those of more "pristine" reefs studied elsewhere in the western Indian Ocean, and have not decreased from historical pre-bleaching studies undertaken elsewhere in southern Madagascar. This observation suggests not only that the levels of bleaching and subsequent reef degradation in Andavadoaka have not yet been sufficient alone to produce the catastrophic decreases in fish biodiversity observed elsewhere (Cumming $e$ al. 2000; Bruno et al. 2001) but also that direct caused a reduction of fish species richness in the Andavadoaka region.

At a local level, however, there are distinct differences in species richness between the four reef types studied in Andavadoaka, which may be due, in part, to fishing pressure. Shallow reefs (shallow barrier fore-reef and inshore fringing forereefs) have been heavily degraded by bleaching events and show lower numbers of species and lower abundance of species than deeper sites (deep barrier fore-reef and deep patch reef). In the event of intensified anthropogenic or increased natural and exploitation, direct reef damage by commercial trawlers, or further severe or prolonged bleaching event), the current low coral cover and poor structural complexity of the region's reefs may make ecosystem function. In light of current growth rates of coastal populations, and predictions of continued increases in sea surface temperature as a result of global climate change (West \& Salm, 2003), it is likely that stresses on southern Madagascar's ree environments will increase, making them more anthropogenic impacts from fishing have not yet climatic stresses on the region's reefs (for example a change in fishing gear technology, increase in them vulnerable to a collapse in fish populations and 
vulnerable to the potentially severe detrimental effects associated with artisanal fisheries (Watson \& Ormond, 1994)

Acknowledgements: The authors would like to thank: Dr Man Wai Rabenavanana of the Institut Halieutique et des Sciences Marines (University of Toliara), and the staff and volunteers of Blue Ventures Conservation, for support at the study site; Gerry Allen for assistance with species identification; and Sophie Benbow and Andrew Cooke for comments made on the manuscript.

\section{REFERENCES}

Allen, G. \& Werner, T. (2002) Coral reef fish assessment in the 'coral triangle' of southeastern Asia. Environmental Biology of Fishes 65: 202214

Battistini, R (1960) Quelques aspects de la morphologie du littoral Mikea (Cote Sud-Ouest de Madagascar) Cahiers Océanogr. XII, 8: $548-571$.

Bellwood, D.R. \& Hughes, T.P. (2001) Regional-scale assembly rules and biodiversity of coral reefs. Science 292: 1532-1534.

Bellwood, D.R., Huguges, T.P., Folke, C., Nyström, M. (2004) Confronting the coral reef crisis. Nature 429: $827-833$.

Bruno, J.F., Siddon, C.E.,Witman, J.D., Colin, P.L. \& Toscano, M.A. (2001) El Nino related bleaching in Palau, Western Caroline Islands. Coral Reefs 20: $127-136$

Chabanet, P. (1994) Etude des relations entre les peuplements benthiques et les peuplement ichtyologiques sur le complexe récifal de StGilles La Saline à l'île de La Réunion. Thèse Environ. Marin, Univ. Aix-Marseille III, 235 pp. + annexes.

Chabanet, P. (2002) Coral reef fish communities of Mayotte (Western Indian Ocean) two years afte the bleaching event. Mar. Fresh. Wat. Res. 53 107-113.

Chabanet, P. \& Durville, P. (2005) Reef fish inventory of Juan de Nova's Natural Park (Western Indian Ocean). Western Indian Ocean Journal of Marine Science 4: 145 -162.

Clausade, M., Gravier, N., Picard, J., Pichon, M Thomassin, B., Vasseur, P., Vivien, M. \& Weydert, P. (1971) Coral reef morphology in the vicinity of Tuléar (Madagascar); contribution to a coral reef terminology. Tethys Suppl. 2: 74

Cooke, A. (2003) Marine and coastal ecosystems of Madagascar. In: "The Natural History of
Madagascar". Goodman, S.M. \& Benstead, J.P. (Eds.) University of Chicago Press: 179-209.

Cooke, A., Ratomahenina O. \& Ranaivosoin E. (2000) Madagascar. In: "Seas at the Millenium" Sheppard C.R.C. (Ed.) Elsevier Science Press: $113-131$.

Cumming, R.L., Lovell, E.R. \& Hay, C. (2000) Geographic extent of the 2000 coral bleaching event in Fiji in relation to water temperature. 9th Int. Coral Reef Symp., Bali (abstract).

Durville, P., Chabanet, P. \& Quod, J.P. (2003) Visual census of the reef fishes in the natural reserve of the Glorieuses Islands (Western Indian Ocean). Western Indian Ocean J. Mar. Sci. 2: 95-104.

English, S., Wilkinson, C. \& Baker, V. (1997) Survey Manual for Tropical Marine Resources, 2nd Edition. Australian Institute of Marine Science (Townsville).

Fowler, J. (1987) The Development of sampling strategies for population studies of coastal reef fishes. A case study. Coral Reefs 6: 49-58.

Gonzalez-Gandara, C., Membrillo-Venegas, N., Nunez-Lara, E. \& Arias-Gonzalez, J.E. (1999) The relationship between fish and reefscapes in the Alacranes Reef, Yucatan, Mexico: A preliminary trophic functioning analysis. Vie et Milieu-Life and Env. 49 (4): 275-286.

Graham, N.A.J., Wilson, S.K., Jennings, S., Polunin, N.V.C., Bijoux, J.P. \& Robinson, J. (2006) Dynamic fragility of oceanic coral reef ecosystems. Dynamic fragility of oceanic coral reef ecosystems.
Proceedings of the National Academy of Sciences Proceedings of the National Acadery

Gratwicke, B. \& Speight, M.R. (2005) Effects of habitat complexity on Caribbean marine fish
hatcke, B. \& Speight, M.R. (2005) Effects of assemblages. Mar. Eco. Prog. Ser. 292: 301-310.

Harmelin-Vivien, M.L. (1977) Ecological Distribution of the fishes on the outer slope of Tulear reef (Madagascar) Proc. 3rd Int. Coral Reef Symp., Miami: 289 - 295.

Harmelin-Vivien, M.L. (1979) Ichtyofaune des récifs coralliens en France Outre-Mer. ICRI. Doc Secrétariat d'Etat à l'Outre-Mer et Ministère de l'Aménagement du Territoire et de l'Environment. $136 \mathrm{pp}$.

Harmelin-Vivien, M.L. (1992) Impact des activités humaines sur les peuplements ichtyologiques des récifs coralliens de Polynésie français. Cybium 16: $279-289$.

Hiatt, W.R. \& Strasburg, D.W. (1960) Ecological relationship of the fish fauna on coral reefs of the Marshall Islands. Ecol. Monogr. 30: 65-127.

Hobson, E.S. (1974) Feeding relationships of Teleostean fishes on coral reefs in Kona, Hawail. Fish Bull. 72: 915-931.
Hughes, T.P., Bellwood, D.R., Connolly, S.R. (2002) Biodiversity hotspots, centres of endemicity, an the conservation of coral reefs. Ecology Letters 5: $775-784$.

Jones, P.J., McCormick, M.I., Srinivasan, M. \& Eagle, J. V. (2004) Coral decline threatens fish biodiversity in marine reserves. Proceedings of the National Academy of Sciences 101(21): 8251-8253.

Maugé, L.A. (1967) Contribution préliminaire à l'inventaire ichthyologique de la région de Tuléar. Rev. Trav. Sta. Endoume, Marseille, Fasc. Hor Rev. Trav. Sta. Endoume,
série, suppl. 7: 101-132.

McClanahan, T. (1994) Kenyan coral reef lagoon fish effects of fishing, substrate complexity, and se urchins. Coral Reefs 13(4): 231-241.

McGehee, M. A. (1994) Correspondence between assemblages of coral reef fishes and gradients of water motion, depth, and substrate size of Puerto Rico. Marine Ecology Progress Serie 105: 243-255 .

McVean, A., Hemery, G., Walker, R. \& Ralisaona, B. (2005) Traditional sea cucumber fisheries in South-West Madagascar: a case-study of two villages. SPC Beche-de-mer Information Bulletin 21: 15-18.

Pichon, M. (1972) The coral reefs of Madagascar. In: "Biogeography and ecology of Madagascar". Richard-Vindard, G. \& Battistini, R. (Eds]. Monogr. Biol., Junk: $367-410$.
Pichon, M. (1974) Free living scleractinian coral communities in the coral reefs of Madagascar Proc 2nd Int. Coral Reef Symp., Manila 2:173181

Randall, J.E. (1998) Zoogeography of shore fishes of the Indo-Pacific region. Zool. Stud. 37: 227-268.

Sala, E. \& Knowlton, N. (2006) Global marine biodiversity trends. Annual Review of Environment and Resources 31: 93-122.

Wantiez, L., Thollot, P., Kulbicki, M. (1997) Effects of marine reserves on coral reef fish communities from five islands in New Caledonia. Coral Reefs 16(4): 215-224.

Watson, M. \& Ormond, R.F.G. (1994) Effect of an artisanal fishery on the fish and urchin population of a Kenyan coral reef Mar. Ecol-Prog. Ser. 109: 115-129.

West, J.M. \& Salm, R.V. (2003) Resistance and Resilience to Coral Bleaching: Implications for Coral Reef Conservation and Management Cons. Bio. 17: 956-967.

Williams, D.M. (1991) Patterns and processes in the distribution of coral reef fishes. In Sale, P.F. (ed) The ecology of fishes on coral reefs. Elsevier Science \& Technology, San Diego, 1991.

Worm, B., Barbier, E.B., Beaumont, N., Duffy, J.E., Folke, C., Halpern, B.S., Jackson, J.B.C., Lotze, H.K., Micheli, F., Palumbi, S.R., Sala, E., Selkoe, K.A., Stachowicz, J.J. Watson, R. (2006) Impacts of Biodiversity Loss on Ocean Ecosystem Services. Science 314(5800): 786-790. 